\title{
Liposomal Nanovesicles For Efficient Encapsulation Of Staphylococcal Antibiotics
}

Azucena Gonzalez Gomez, ${ }^{a}$ Saifuddin Syed, ${ }^{a}$ Kenji Marshall, ${ }^{a}$ Zeinab Hosseinidoust ${ }^{a, b *}$

${ }^{a}$ Department of Chemical Engineering, McMaster University, Hamilton, ON, L9S 8L7, Canada b Michael DeGroote Institute for Infectious Disease Research, McMaster University, Hamilton, Ontario, L98 4L8, Canada

*Corresponding Author. Phone: (905) 525-9140; email: doust@mcmaster.ca 
Supporting Information

Calculation of estimated encapsulation efficiency:

The efficiency calculation was done for FAT-Vanco liposomes with an average size of $215 \mathrm{~nm}$ and a polydispersity index (PDI) of 0.246 (Figure S1). The formula used was:

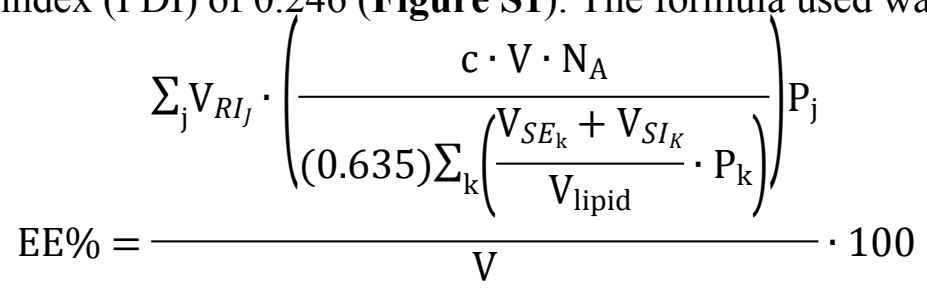

$\mathrm{EE}=$ encapsulation efficiency

$\mathrm{c}=$ lipid concentration

$\mathrm{V}=$ volume of liquid added

$\mathrm{NA}=$ Avogadro's constant

$\mathrm{VRI}=$ volume ring internal

$\mathrm{VSE}=$ volume sphere external

VSI = volume sphere internal

Vlipid = volume lipid

$\mathrm{P}=$ liposome distribution

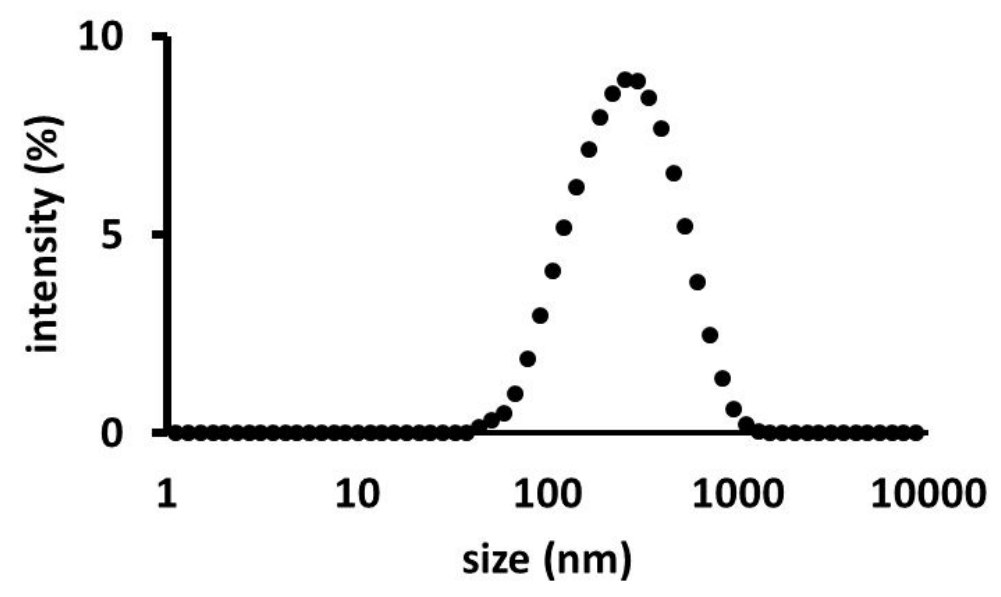

Figure S1. Size distribution of FAT-Vanco liposomes, $18 \%$ solubility in water, obtained by dynamic light scattering. 


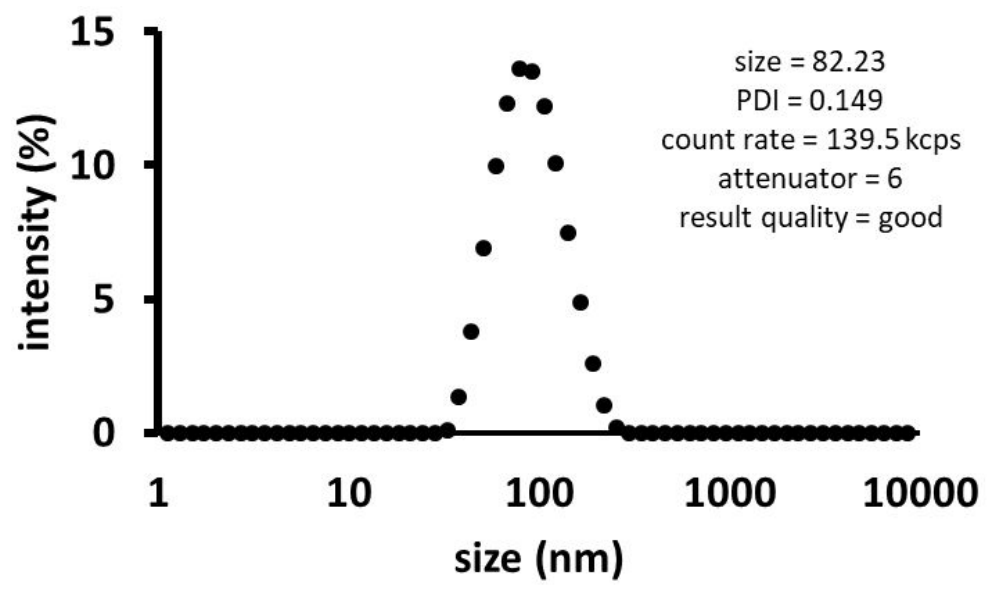

Figure S2. DLS results of teicoplanin liposomes created by REV with $80 \%$ of teicoplanin's solubility in water. 